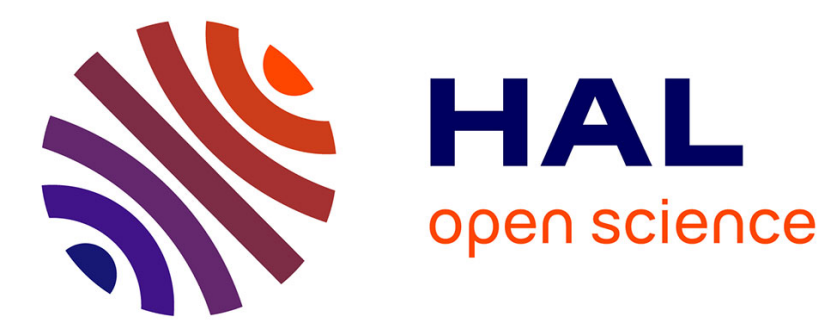

\title{
Drag Minimization of the Cylinder Wake by Trust-Region Proper Orthogonal Decomposition
}

Michel Bergmann, Laurent Cordier, Jean-Pierre Brancher

\section{To cite this version:}

Michel Bergmann, Laurent Cordier, Jean-Pierre Brancher. Drag Minimization of the Cylinder Wake by Trust-Region Proper Orthogonal Decomposition. Active Flow Control 2006, Sep 2006, Berlin, France. pp.309-324, 10.1007/978-3-540-71439-2_19. inria-00338822

\section{HAL Id: inria-00338822 \\ https://hal.inria.fr/inria-00338822}

Submitted on 14 Nov 2008

HAL is a multi-disciplinary open access archive for the deposit and dissemination of scientific research documents, whether they are published or not. The documents may come from teaching and research institutions in France or abroad, or from public or private research centers.
L'archive ouverte pluridisciplinaire HAL, est destinée au dépôt et à la diffusion de documents scientifiques de niveau recherche, publiés ou non, émanant des établissements d'enseignement et de recherche français ou étrangers, des laboratoires publics ou privés. 


\title{
Drag Minimization of the Cylinder Wake by Trust-Region Proper Orthogonal Decomposition
}

\author{
Michel Bergmann, Laurent Cordier, and Jean-Pierre Brancher \\ LEMTA, UMR 7563 (CNRS - INPL - UHP) \\ 2 , avenue de la forêt de Haye \\ BP 160 - 54504 Vandoeuvre cedex, France
}

\begin{abstract}
Summary
In this paper we investigate the optimal control approach for the active control of the circular cylinder wake flow considered in the laminar regime $(R e=200)$. The objective is the minimization of the mean total drag where the control function is the time harmonic angular velocity of the rotating cylinder. When the Navier-Stokes equations are used as state equations, the discretization of the optimality system leads to large scale discretized optimization problems that represent a tremendous computational task. In order to reduce the number of state variables during the optimization process, a Proper Orthogonal Decomposition (POD) Reduced-Order Model (ROM) is then derived to be used as state equation. Since the range of validity of the POD ROM is generally limited to the vicinity of the design parameters in the control parameter space, we propose to use the Trust-Region Proper Orthogonal Decomposition (TRPOD) approach, originally introduced by Fahl (2000), to update the reduced-order models during the optimization process. Benefiting from the trust-region philosophy, rigorous convergence results guarantee that the iterates produced by the TRPOD algorithm will converge to the solution of the original optimization problem defined with the Navier-Stokes equations. A lot of computational work is indeed saved because the optimization process is now based only on low-fidelity models. The key enablers to an accurate and robust POD ROM for the pressure and velocity fields are the extension of the POD basis functions to the pressure data, the introduction of a time-dependent eddy-viscosity estimated for each POD mode as the solution of an auxiliary optimization problem, and the inclusion in the POD ROM of different non-equilibrium modes. When the TRPOD algorithm is applied to the wake flow configuration, this approach converges to the minimum predicted by an open-loop control approach and leads to a relative mean drag reduction of $30 \%$ for reduced numerical costs (a cost reduction factor of 1600 is obtained for the memory and the optimization problem is solved approximately 4 times more quickly).
\end{abstract}




\section{Introduction}

\subsection{Managing the Use of Approximation Models in Optimization}

During the last decade, the optimal control theory [1] has emerged as a new approach to solve active flow control and aerodynamic shape design problems. Indeed, these problems can be reduced [2] to the minimization or maximization of an objective functional $\mathcal{J}$ (drag or lift coefficients, concentration of pollutant, emitted noise, mixing ... ) according to $n$ control or design parameters $\boldsymbol{c}=\left(c_{1}, c_{2}, \cdots, c_{n}\right)$ (unsteady blowing/suction velocities, heat flows, ...) under some constraints (Navier-Stokes equations, geometric constraints ....). Roughly, these optimization problems can be solved by two different class of numerical methods, on the one hand, the methods of descent type which at least require an approximation of the gradient of the objective functional, and, on the other hand, the stochastic methods whose principle consists in studying the evolution of a population of potential solutions during successive generations (genetic algorithms, simplex method ....). Whatever the specific class of numerical methods considered, the computational costs (CPU and memory) related to the resolution of optimization problems are so important that they become unsuited to the applications of flow control. This situation is even worse in an optimization setting where the governing equations need to be solved repeatedly, or in closed-loop control problems, for which the controller needs to determine his action in real time. Consequently, an alternative approach is necessary.

In this communication, we propose to solve the aforementioned problem of optimization by an optimal control approach in which the Navier-Stokes equations - called high-fidelity model in the multidisciplinary optimization literature - are replaced by a Proper Orthogonal Decomposition (POD) ReducedOrder Model (ROM) - low-fidelity model - of the dynamics for the controlled flow. The POD was originally introduced in Turbulence [3] as an unbiased method of extraction of the Coherent Structures widely known to exist in a turbulent flow. Essentially, this technique leads to the evaluation of POD functions that define a flow basis, optimal in an energetic sense. Thereafter, these POD modes can be used through a Galerkin projection on the NavierStokes equations to derive a POD ROM of the controlled flow [4]. The POD basis is determined a posteriori using experimental or numerical data previously obtained for the configuration under study. In first approximation, the POD can be viewed as a method of information compression. Essentially, the POD algorithm try to remove "redundant" information (if any) from the data base. As a consequence, the ability of POD modes to approximate any state of a complex system is totally dependent of the information originally contained in the snapshot set used to generate the POD functions. Then, despite the energetic optimality of the POD modes, it seems difficult to build once for all, at the beginning of the optimization process, a POD ROM able to approximate correctly the different controlled states encountered by the flow 
along the optimal path (see the discussion in [5]). Some kind of reactualization of the POD basis during the optimization process seems essential, the main difficulty consisting in determining the moment when a new resolution of the Navier-Stokes equations is necessary to evaluate a new POD basis. Thereafter, we will use a specific adaptive method called Trust-Region POD (TRPOD) to update the reduced-order models during the optimization process. This approach, originally introduced in [6], couples a trust-region method of optimization and reduced-order models based on POD (see Sect. 2). The principal advantages of this approach are, on the one hand, that the radius of the trust-region corresponding to the POD ROM does not have to be fixed by the user, but is evaluated automatically during the optimization process, and that on the other hand, there are results of convergence proving that the iterates produced by the TRPOD algorithm will converge to the solution of the original optimization problem defined with the Navier-Stokes equations.

\subsection{A Prototype of Massively Separated Flows: the Cylinder Wake Flow}

In this study, we are interested to control the unsteady wake flow downstream from a circular cylinder (Fig. 1). The objective is the mean drag minimization

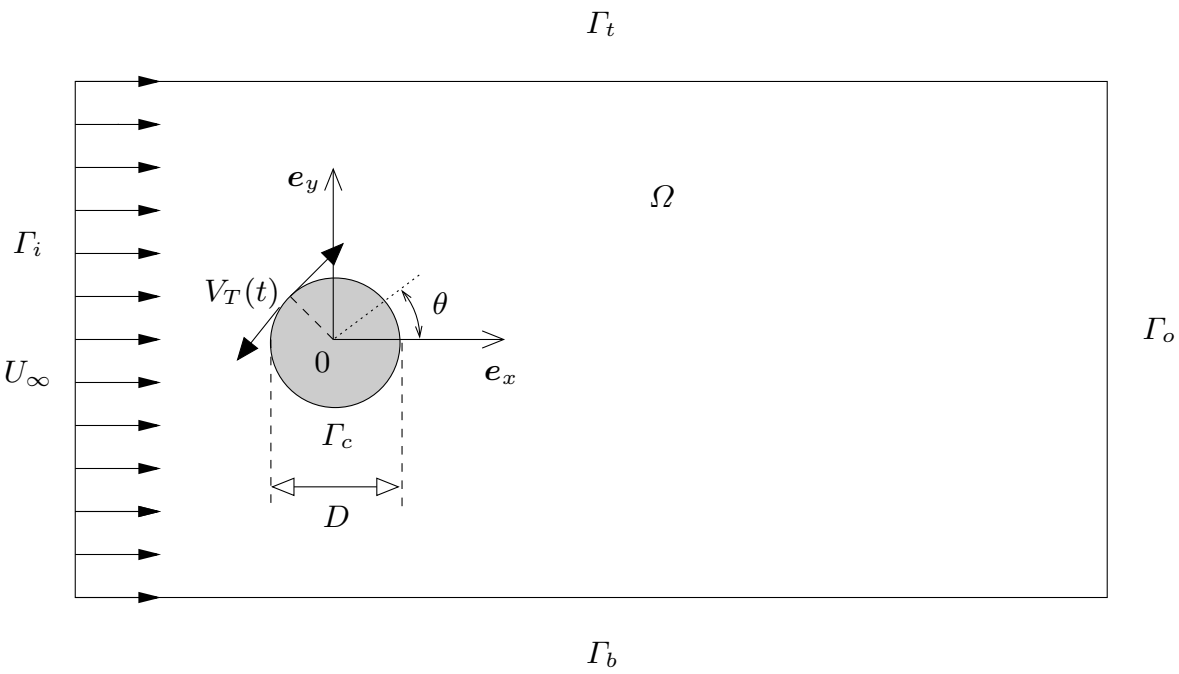

Figure 1 Controlled flow configuration.

of the wake flow by rotary oscillation of the cylinder. The flow is considered as incompressible and the fluid is supposed to be viscous and Newtonian. Wake flows dynamics are characterized [7] by the Reynolds number $R e$ and by the 
natural Strouhal number $S t_{n}$ at which vortices are shed in the wake of the cylinder (Fig. 8(a)). Traditionally, the Reynolds number is defined as $R e=$ $U_{\infty} D / \nu$ where $D$ is the cylinder diameter ( $R$ is the corresponding radius), $U_{\infty}$ the uniform velocity of the incoming flow and $\nu$ the kinematic viscosity of the fluid. As for the natural Srouhal number, the common definition is $S t_{n}=$ $f D / U_{\infty}$ where $f$ is the frequency characteristic of the periodic behavior of the flow. The cylinder wake is considered in the laminar regime $(R e=200)$. The rotary control is characterized by the non dimensional velocity $\gamma(t)$ defined as the ratio of the tangential velocity $V_{T}$ to the upstream velocity $U_{\infty}$ i.e. $\gamma(t)=V_{T}(t) / U_{\infty}$. For $\gamma=0$, the flow is naturally said uncontrolled. Contrary to the case considered in [5] where no particular assumption was done on the variation of the control function, $\gamma(t)$ is hereafter sought as an harmonic function of the form:

$$
\gamma(t)=A \sin \left(2 \pi S t_{f} t\right)
$$

where the amplitude $A$ and the forcing Strouhal number $S t_{f}$ correspond to two degrees of freedom for the control. The optimal control theory is then used to determine the control vector $\boldsymbol{c}=\left(A, S t_{f}\right)^{T}$ which minimizes the mean time drag coefficient of the wake. For a circular cylinder, this quantity estimated over a finite horizon $T$ equal to a few vortex shedding periods writes:

$$
\begin{aligned}
\left\langle C_{D}\right\rangle_{T} & =\frac{1}{T} \int_{0}^{T} \int_{0}^{2 \pi} 2 p n_{x} R d \theta d t \\
& -\frac{1}{T} \int_{0}^{T} \int_{0}^{2 \pi} \frac{2}{R e}\left(\frac{\partial u}{\partial x} n_{x}+\frac{\partial u}{\partial y} n_{y}\right) R d \theta d t
\end{aligned}
$$

where $n_{x}$ and $n_{y}$ are the projections of the external normal vector $\boldsymbol{n}$ onto the cartesian basis vectors $\boldsymbol{e}_{x}$ and $\boldsymbol{e}_{y}$ respectively, and $\theta$ is an angle defining the curvilinear coordinate of a point on $\Gamma_{c}$ (see Fig. 1). Furthermore, let us recall (see [8] for example), that in the supercritical regime of the wake flow, every mean quantity consists of two terms, the basic flow i.e., the unstable, steady, symmetric flow, and the mean flow correction which is due to the vortex shedding. Consequently, the mean time drag coefficient $\left\langle C_{D}\right\rangle_{T}$ writes:

$$
\left\langle C_{D}\right\rangle_{T}=C_{D}^{\text {basic }}+C_{D}^{0},
$$

where $C_{D}^{\text {basic }}$ and $C_{D}^{0}$ represent the drag of the basic flow and the mean flow correction respectively (see Fig. 2). Of course, at a given Reynolds number, the contribution of the basic flow to drag cannot be modified. Then controlling the wake flow by rotary oscillations can only reduce the contribution of the mean flow correction to drag. If we consider as in [8] that the drag of the mean flow correction field can be only positive, the minimal value of drag that can be obtained under periodic forcing conditions is that corresponding to the basic flow. In conclusion, this flow would be thus a natural 'desired' field in a flow tracking procedure of optimization. 


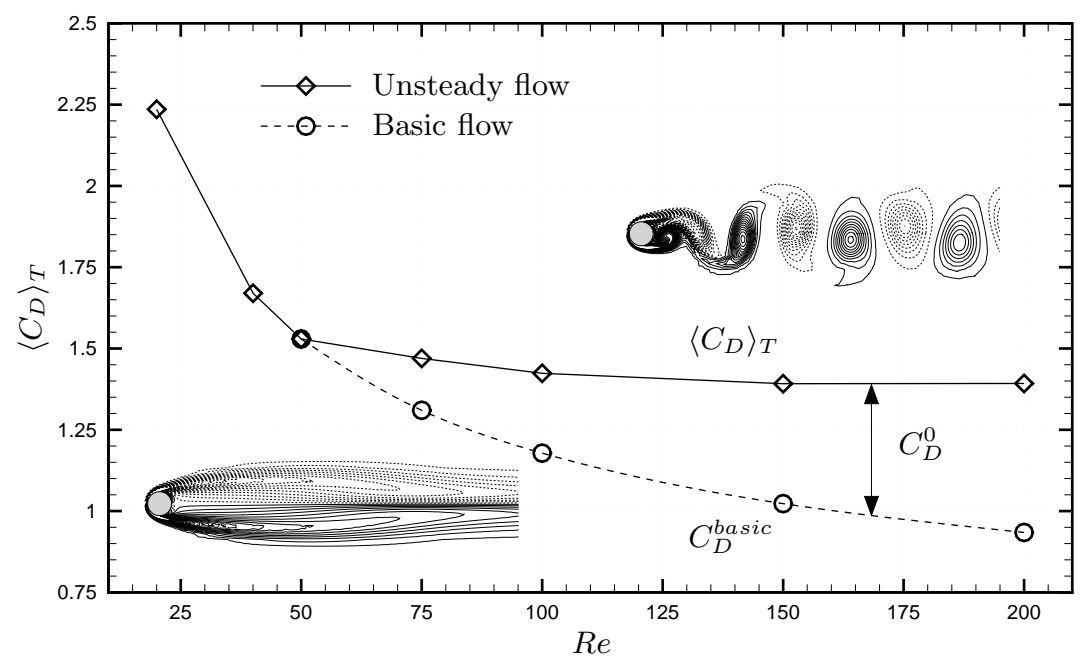

Figure 2 Variation with the Reynolds number of the mean drag coefficient. Contributions and corresponding flow patterns of the basic flow and unsteady flow.

In order to validate a posteriori the control law obtained with the TRPOD algorithm, an open-loop control study is first performed numerically. The contours of the mean temporal drag coefficient, estimated over approximately 6 periods of vortex shedding after the transients have died out, are visualized in Fig. 3 in the space spanned by the forcing parameters $A$ and $S t_{f}$.

\section{Optimization by Trust-Region Methods and POD Reduced-Order Models}

In this section, only the principle of the Trust-Region Proper Orthogonal Decomposition approach for flow control is exposed. For all the details of the algorithms and in particular the proofs of convergence, the reader is referred to $[6,11]$.

We consider that the flow control problem discussed in Sect. 1.1 can be formulated as an unconstrained optimization problem

$$
\min _{\boldsymbol{c} \in \mathbb{R}^{n}} \mathcal{J}\left(\phi_{N S}(\boldsymbol{c}), \boldsymbol{c}\right)
$$

where $\mathcal{J}: \mathbb{R}^{m} \times \mathbb{R}^{n} \mapsto \mathbb{R}$ represents the objective functional and where $\phi_{N S}$ and $\boldsymbol{c}$ respectively represent the state variables obtained by numerical resolution of the state equations and the control variables. The subscript $N S$ 


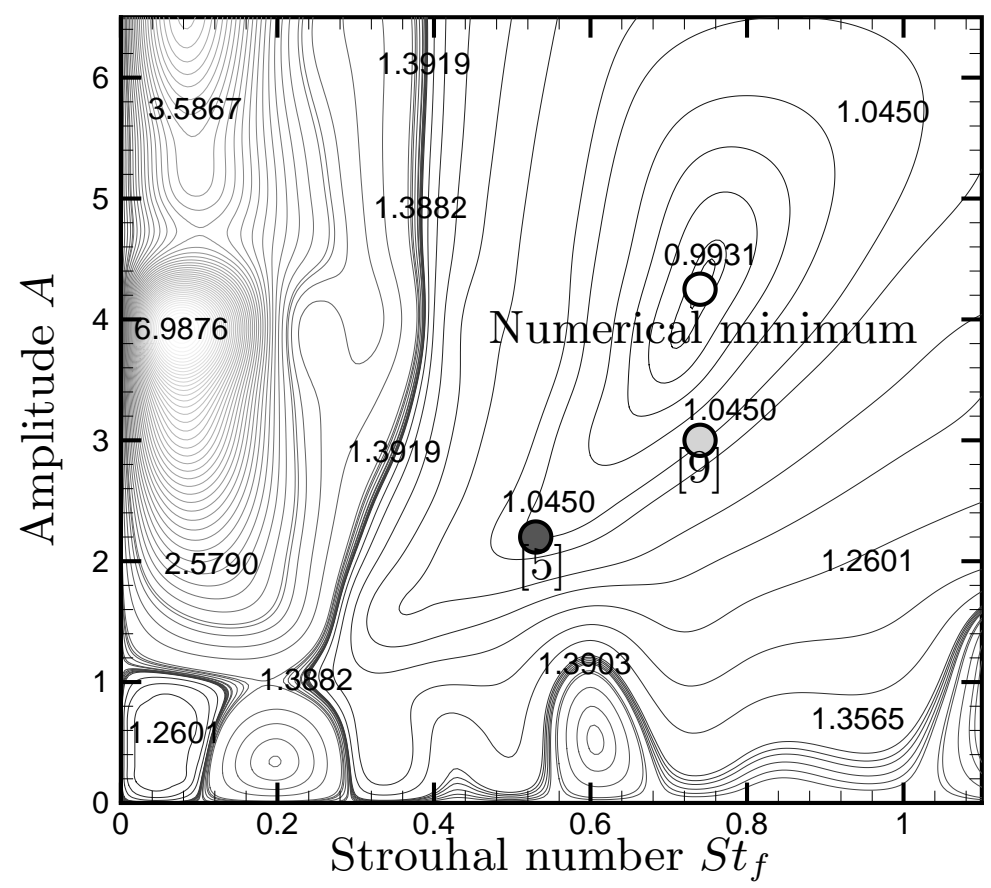

Figure 3 Variation of the mean drag coefficient with $A$ and $S t_{f}$ at $R e=200$. Numerical minimum: $\left(A_{\min }, S t_{f_{\min }}\right)=(4.25,0.74) ;[9]:\left(A, S t_{f}\right)=(3 ., 0.75) ;[10]$ (not shown): $\left(A, S t_{f}\right)=(3.25,1.13) ;[5]:\left(A, S t_{f}\right)=(2.2,0.53)$. 
means that the state equations which connect the control variables $\boldsymbol{c}$ to the state variables are the Navier-Stokes equations. Since an accurate computation of the state variables $\phi$ for given $\boldsymbol{c}$ is computationally expensive when the Navier-Stokes equations are used as the state equations, the evaluation of $\mathcal{J}$ during the solution of the optimization process (3) is computationally expensive. A reduction of numerical cost can be achieved by employing a POD ROM as the state equation. In such a way an approximate solution $\phi_{P O D}$ of the state variables $\phi$ is obtained and the optimization problem (3) is then replaced by a succession of subproblems of the form

$$
\min _{\boldsymbol{c} \in \mathbb{R}^{n}} \mathcal{J}\left(\phi_{P O D}(\boldsymbol{c}), \boldsymbol{c}\right) .
$$

Usually, a POD ROM is constructed for a specific flow configuration, e.g., for an uncontrolled flow or for a flow altered by a specified control. Therefore, the range of validity of a given POD ROM is generally restricted to a region located in the vicinity of the design parameters in the control parameter space, the so-called trust-region. It is then necessary to update the POD ROM during the iterative process, the crucial point being to determine when such a reactualization must take place. Let $\Delta_{k}>0$ be the trust-region radius and $\boldsymbol{c}_{k}$ be the control parameters obtained at an iterate $k$ of the optimization process. To evaluate the function $\mathcal{J}\left(\phi_{N S}\left(\boldsymbol{c}_{k}\right), \boldsymbol{c}_{k}\right)$, it is necessary to determine the variables $\phi_{N S}\left(\boldsymbol{c}_{k}\right)$. These variables are obtained by resolution of the highfidelity model, the Navier-Stokes equations. Then, we compute snapshots that correspond to the flow dynamics forced by $\boldsymbol{c}_{k}$. These snapshots form the input ensemble necessary [3] to generate a POD basis $\left\{\boldsymbol{\Phi}_{i}^{k}\right\}_{i=1, \ldots, N_{P O D}}$ (here, $N_{P O D}$ corresponds to the number of POD modes). This POD basis can then be used via a Galerkin projection of the Navier-Stokes equations onto the POD eigenvectors to derive a POD ROM for $\boldsymbol{c}_{k}[11,5]$. After integration in time of this POD ROM, the state variables $\phi_{P O D}\left(\boldsymbol{c}_{k}\right)$ are estimated, and thus the function $\mathcal{J}\left(\phi_{P O D}\left(\boldsymbol{c}_{k}\right), \boldsymbol{c}_{k}\right)$ is evaluated. Since this POD ROM can be employed for an optimization cycle, we define

$$
m_{k}\left(\boldsymbol{c}_{k}+\boldsymbol{s}_{k}\right)=\mathcal{J}\left(\phi_{P O D}\left(\boldsymbol{c}_{k}+\boldsymbol{s}_{k}\right), \boldsymbol{c}_{k}+\boldsymbol{s}_{k}\right),
$$

as a model function for

$$
f\left(\boldsymbol{c}_{k}+\boldsymbol{s}_{k}\right)=\mathcal{J}\left(\phi_{N S}\left(\boldsymbol{c}_{k}+\boldsymbol{s}_{k}\right), \boldsymbol{c}_{k}+\boldsymbol{s}_{k}\right),
$$

on the trust-region $\left\|s_{k}\right\| \leq \Delta_{k}$ around $\boldsymbol{c}_{k}$.

One is then brought to solve approximately ${ }^{1}$ the corresponding trustregion subproblem defined as

$$
\min _{\boldsymbol{s} \in \mathbb{R}^{n}} m_{k}\left(\boldsymbol{c}_{k}+\boldsymbol{s}\right), \quad \text { s.t. }\|\boldsymbol{s}\| \leq \Delta_{k} .
$$

\footnotetext{
${ }^{1}$ Following the trust-region philosophy [12], it is sufficient to compute a trial step $s_{k}$ that achieves only a certain amount of decrease for the model function.
} 
In order to estimate the quality of the presumed next control parameters $\boldsymbol{c}_{k+1}=\boldsymbol{c}_{k}+\boldsymbol{s}_{k}$ where $\boldsymbol{s}_{k}$ is an approximate solution of (7), we compare the actual reduction in the true objective, ared $_{k}=f\left(\boldsymbol{c}_{k}+\boldsymbol{s}_{k}\right)-f\left(\boldsymbol{c}_{k}\right)$, to the predicted reduction obtained with the model function $\operatorname{pred}_{k}=m_{k}\left(\boldsymbol{c}_{k}+\right.$ $\left.\boldsymbol{s}_{k}\right)-m_{k}\left(\boldsymbol{c}_{k}\right)$. Essentially, it is this comparison that gives a measure for the current models prediction capability. If the trial step $s_{k}$ yields to a satisfactory decrease in the original objective functional in comparison to the one obtained by the model function, the iteration is successful, the trial step $\boldsymbol{s}_{k}$ is accepted and the model $m_{k}$ is updated i.e. a new POD ROM is derived that incorporates the flow dynamics as altered by the new $\operatorname{control}^{2} \boldsymbol{c}_{k+1}$. Furthermore, if the achieved decrease in $f$ indicates a good behavior of the model $m_{k}$, the trust-region radius $\Delta_{k}$ can be increased. Now, if there is a limited predicted decrease compared to the actual decrease, we have the possibility to decrease slightly the value of the trust-region radius. For unsuccessful iterations, the trial step $s_{k}$ is not accepted, the trust-region radius $\Delta_{k}$ is decreased and the trust-region subproblem (7) is solved again within a smaller trust-region. With the contraction of the trust-region it is more likely to have a good approximation to the true objective functional with the POD ROM. The corresponding TRPOD algorithm is schematically described in Fig. 4.

\section{Drag Minimization of the Cylinder Wake Flow by the TRPOD Algorithm}

The objective of this section is to implement the TRPOD approach presented in Sect. 2 for minimizing the mean drag coefficient of the cylinder wake flow.

\subsection{A Robust POD Surrogate for Drag Function}

In order to simplify the future notations, one introduces the drag operator $\mathcal{C}_{\mathcal{D}}$ defined as:

$$
\begin{aligned}
\mathcal{C}_{\mathcal{D}}: \mathbb{R}^{3} & \mapsto \mathbb{R} \\
\boldsymbol{U} & \mapsto 2 \int_{0}^{2 \pi}\left(p n_{x}-\frac{1}{R e} \frac{\partial u}{\partial x} n_{x}-\frac{1}{R e} \frac{\partial u}{\partial y} n_{y}\right) R d \theta,
\end{aligned}
$$

where $\boldsymbol{U}=(u, v, p)^{T}$ denotes the vector corresponding to the velocity and pressure fields. By definition, $\mathcal{C}_{\mathcal{D}}(\boldsymbol{U})=C_{D}(t)$ where $C_{D}$ represents the instantaneous drag coefficient. The velocity component $u$ and pressure $p$ present in the relation (8) can be obtained either by resolution of the NavierStokes equations, or by estimation using a POD ROM. In this study, a special

\footnotetext{
${ }^{2}$ Since a new snapshot ensemble is available, a new POD basis can then be determined, and finally, a new POD ROM can be derived.
} 
Initialization: $\boldsymbol{c}_{0}$, Navier-Stokes resolution, $\mathcal{J}_{0} . k=0$.

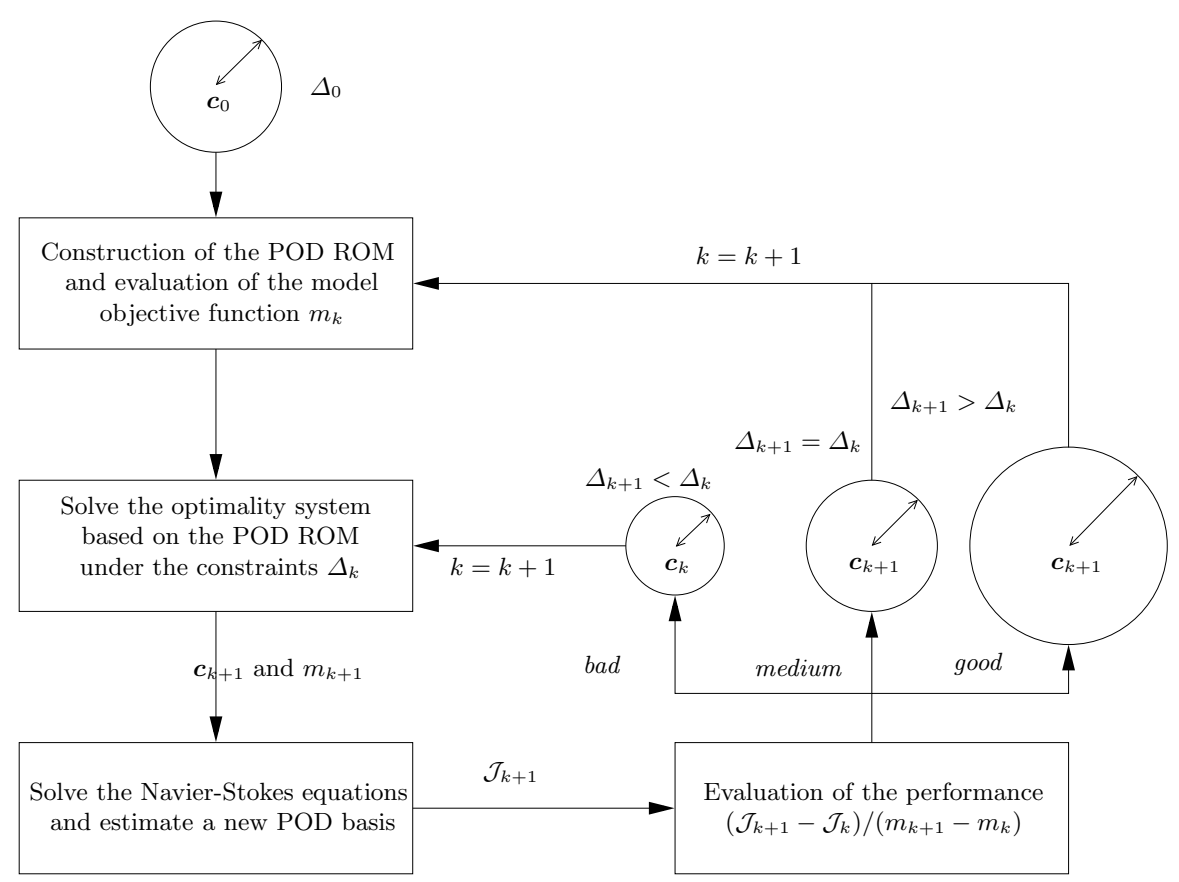

Figure 4 Schematic representation of the TRPOD algorithm. 
care is taken to the development of the POD ROM. First, a POD basis $\boldsymbol{\Phi}_{i}$ representative of the velocity fields $u$ and $v$, as of the pressure field $p$ was determined [11]. Then, to improve the robustness of the POD ROM, the POD basis functions which represent the dynamics of the reference operating condition $\boldsymbol{c}$, were increased by adding $N_{\text {neq }}$ non-equilibrium modes corresponding to new operating conditions, following the procedure described in [13]. In addition, a calibration procedure of the POD-Galerkin system similar to the methods recently introduced in [14] for the pressure model was used to take into account some modelling errors (Galerkin truncation, pressure model,...). Here, this calibration procedure corresponds to the introduction of a timedependent eddy-viscosity estimated for each POD mode as the solution of an auxiliary optimization problem (see [15] for all the details). Finally, the control function method introduced in [16] is used to determine POD basis functions with homogeneous boundary conditions. The velocity and pressure fields can then be expanded into the POD basis functions $\boldsymbol{\Phi}_{i}$ as:

$$
\boldsymbol{U}(\boldsymbol{x}, t)=\underbrace{\sum_{i=0}^{N_{\text {gal }}} a_{i}(t) \boldsymbol{\Phi}_{i}(\boldsymbol{x})}_{\text {Galerkin modes }}+\underbrace{\sum_{i=N_{\text {gal }}+1}^{N_{\text {gal }}+N_{\text {neq }}} a_{i}(t) \boldsymbol{\Phi}_{i}(\boldsymbol{x})}_{\text {non-equilibrium modes }}+\underbrace{\gamma(\boldsymbol{c}, t) \boldsymbol{U}_{c}(\boldsymbol{x})}_{\text {control function }},
$$

where $N_{\text {gal }}$ is the number of Galerkin modes and where $\boldsymbol{U}_{c}$ is called the control function. Mathematically, $\boldsymbol{U}_{c}$ is determined as a solution of the Navier-Stokes equations satisfying specific boundary conditions (the procedure is fully described in $[11,5])$.

The Galerkin projection of the Navier-Stokes equations on the space spanned by the first $N_{g a l}+N_{n e q}+1$ POD modes yields [11] to

$$
\begin{aligned}
\frac{d a_{i}(t)}{d t}= & \sum_{j=0}^{N_{\text {gal }}+N_{\text {neq }}} \mathcal{B}_{i j} a_{j}(t)+\sum_{j, k=0}^{N_{\text {gal }}+N_{\text {neq }}} \mathcal{C}_{i j k} a_{j}(t) a_{k}(t) \\
& +\mathcal{D}_{i} \frac{d \gamma}{d t}+\left(\mathcal{E}_{i}+\sum_{j=0}^{N_{\text {gal }}+N_{\text {neq }}} \mathcal{F}_{i j} a_{j}(t)\right) \gamma(\boldsymbol{c}, t)+\mathcal{G}_{i} \gamma^{2}(\boldsymbol{c}, t)
\end{aligned}
$$

with the following initial conditions:

$$
a_{i}(0)=\left(\boldsymbol{u}(\boldsymbol{x}, 0), \boldsymbol{\Phi}_{i}(\boldsymbol{x})\right) .
$$

The coefficients $\mathcal{B}_{i j}, \mathcal{C}_{i j k}, \mathcal{D}_{i}, \mathcal{E}_{i}, \mathcal{F}_{i j}$ and $\mathcal{G}_{i}$ depend explicitly on $\boldsymbol{\Phi}_{i}$ and $\boldsymbol{U}_{c}$. Their expression could be found in [5].

Let $\phi_{N S}(\boldsymbol{c})=\left(u_{N S}, v_{N S}, p_{N S}\right)^{T}$ represent the state variables obtained by resolution of the Navier-Stokes equations and $\phi_{P O D}(\boldsymbol{c})=\left(u_{P O D}, v_{P O D}\right.$ 
, $\left.p_{P O D}\right)^{T}$ be the corresponding values estimated with the POD ROM (10), the objective functional is

$$
f(\boldsymbol{c})=\mathcal{J}\left(\phi_{N S}(\boldsymbol{c})\right)=\frac{1}{T} \int_{0}^{T} \mathcal{C}_{D}\left(\phi_{N S}(\boldsymbol{c})\right) d t,
$$

and the model function, introduced and justified in [11], is

$$
m_{k}(\boldsymbol{c})=\widetilde{\mathcal{J}}\left(\phi_{P O D}(\boldsymbol{c})\right)=\frac{1}{T} \int_{0}^{T} \sum_{i=0}^{N_{g a l}+N_{n e q}} a_{i}(t) N_{i} d t
$$

where $N_{i}=\mathcal{C}_{D}\left(\boldsymbol{\Phi}_{i}\right)$

These two functions can then be used in a procedure of optimization coupling trust-region methods and POD reduced-order models, following the method presented in Sect. 2.

\subsection{Solution of the Subproblem (7)}

The convergence behavior of trust-region methods for general model functions with inexact gradient information is usually based on a sufficient decrease condition of the objective function (see [12] for example). In [6], these classical results were extended and it was demonstrated that the exact solution of the subproblem (7) is not necessary to prove global convergence of the TRPOD algorithm. Here, because of the low computational costs of solving the POD reduced-order model (10), an optimality system based on the POD ROM is derived (see [2] for example) and solved. By definition, this reduced-order optimality system is a system of three coupled partial differential equations [11] formed by:

1. the state equations (10)

2. the adjoint equations

$$
\begin{aligned}
\frac{d \xi_{i}(t)}{d t}= & -\sum_{j=0}^{N_{g a l}+N_{\text {neq }}}\left(\mathcal{B}_{j i}+\gamma(\boldsymbol{c}, t) \mathcal{F}_{j i}\right) \xi_{j}(t) \\
& -\sum_{j, k=0}^{N_{g a l}+N_{\text {neq }}}\left(\mathcal{C}_{j i k}+\mathcal{C}_{j k i}\right) a_{k}(t) \xi_{j}(t)-\frac{1}{T} N_{i},
\end{aligned}
$$

with terminal conditions :

$$
\xi_{i}(T)=0 .
$$


3. the optimality conditions

$$
\nabla_{\boldsymbol{c}} \widetilde{\mathcal{J}}=\frac{1}{T} \int_{0}^{T}\left(\sum_{i=0}^{N_{\text {gal }}+N_{\text {neq }}} \mathcal{L}_{i}\right) \nabla_{\boldsymbol{c}} \gamma d t
$$

with

$$
\mathcal{L}_{i}=-\frac{d \xi_{i}}{d t} \mathcal{D}_{i}+\xi_{i}\left(\mathcal{E}_{i}+\sum_{j=0}^{N_{g a l}+N_{\text {neq }}} \mathcal{F}_{i j} a_{j}+2 \gamma(\boldsymbol{c}, t) \mathcal{G}_{i}\right)
$$

This system of coupled ordinary differential equations could be solved directly using a "one-shot method". However, due to large storage and CPU costs, an iterative process described in [11] is usually preferred. In this study, the directions of descent are estimated using the Fletcher-Reeves version of the Conjugate Gradient Method [17]. The linear search parameter is computed at each iteration by the backtracking Armijo method [17], in which the length of the step, along each direction of descent, checks the constraint imposed by the trust-region approach. The iterative method is stopped when two following values of the functional $\widetilde{\mathcal{J}}$ are sufficiently close i.e. when $|\Delta \widetilde{\mathcal{J}}(\boldsymbol{a})|$ $=\left|\widetilde{\mathcal{J}}^{(n+1)}(\boldsymbol{a})-\widetilde{\mathcal{J}}^{(n)}(\boldsymbol{a})\right|<10^{-5}$.

\subsection{Numerical Results and Discussion}

As it was discussed for example in [18], a possible drawback of solving a minimization problem with a gradient-based optimization approach is that the algorithm may converge to the global minimum or to some other local minimum of the cost function depending on the relative position of the starting point to the minima. To alleviate this difficulty and evaluate the robustness of the TRPOD algorithm, the optimization process will be initialized starting from several different control parameters $\boldsymbol{c}_{0}=(A ; S t)$ chosen at random in the control parameter space retained for the open-loop control procedure (see Fig. 3). Hereafter, four different initial values are employed: $\boldsymbol{c}_{0}=(1.0,0.2)^{T}$, $\boldsymbol{c}_{0}=(1.0,1.0)^{T}, \boldsymbol{c}_{0}=(6.0,0.2)^{T}$ and $\boldsymbol{c}_{0}=(6.0,1.0)^{T}$.

According to the TRPOD algorithm (see Fig. 4), the radius of the trustregion $\Delta$ is automatically either increased, or decreased during the resolution of the optimization process. Figure 5 represents for the different initial control parameters $\boldsymbol{c}_{0}$, the variations of the values of the forcing amplitude and Strouhal number with respect to the iteration number. When the numerical convergence of the iterative procedure is achieved, the optimal control parameters are $A=4.25$ and $S t_{f}=0.738$. These values of parameters, which entirely define the optimal control law $\gamma_{o p t}(t)$, are obtained in less than ten resolutions of the Navier-Stokes equations, whatever the initial condition considered (a more significant number of iterations is however represented in Fig. 5 to highlight the convergence). As it was expected by the 

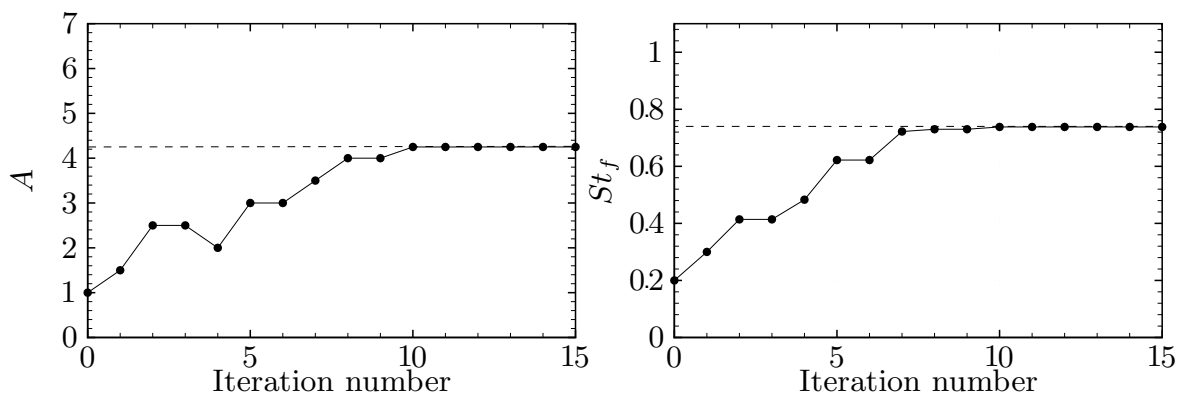

(a) $\boldsymbol{c}_{0}=(1.0,0.2)^{T}$
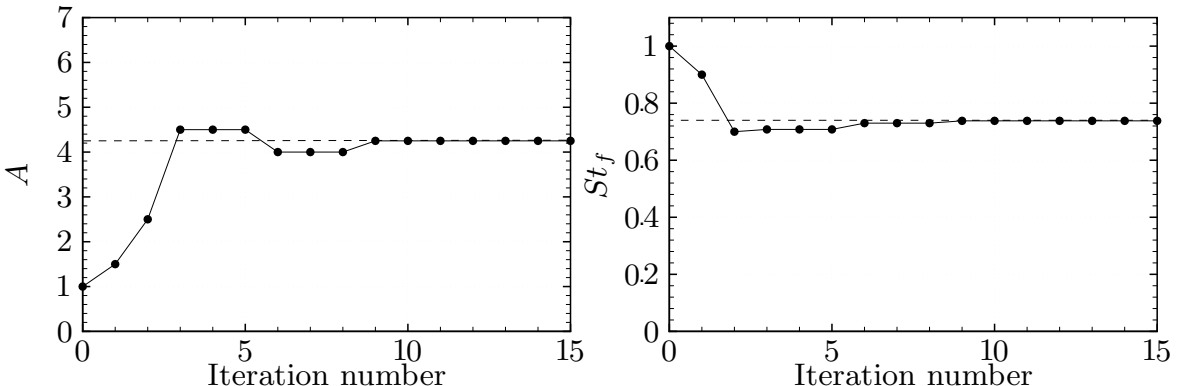

(b) $\boldsymbol{c}_{0}=(1.0,1.0)^{T}$.
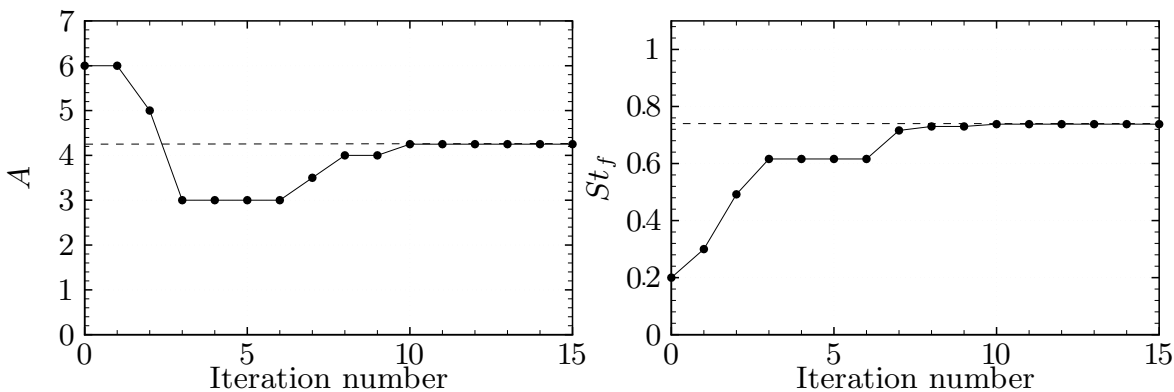

(c) $\boldsymbol{c}_{0}=(6.0,0.2)^{T}$.
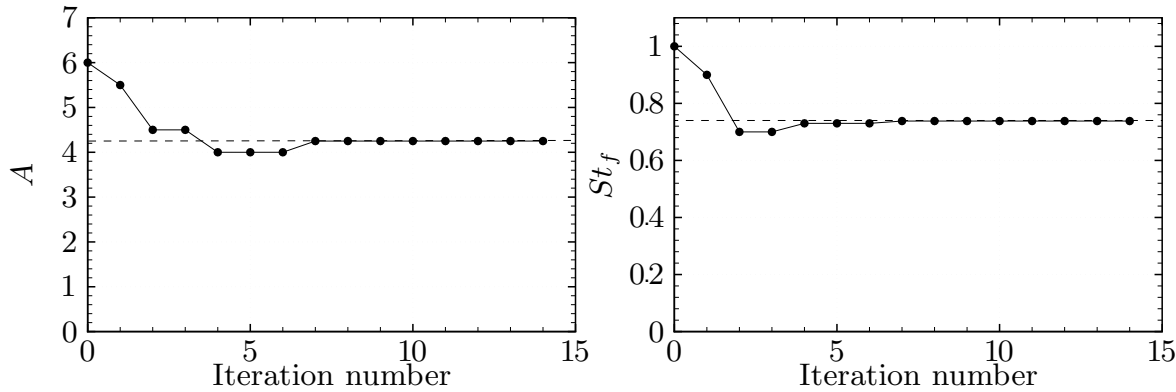

(d) $\boldsymbol{c}_{0}=(6.0,1.0)^{T}$.

Figure 5 Variations of the forcing amplitude (left) and Strouhal number (right) with respect to the iteration number. Results obtained with the TRPOD algorithm. 


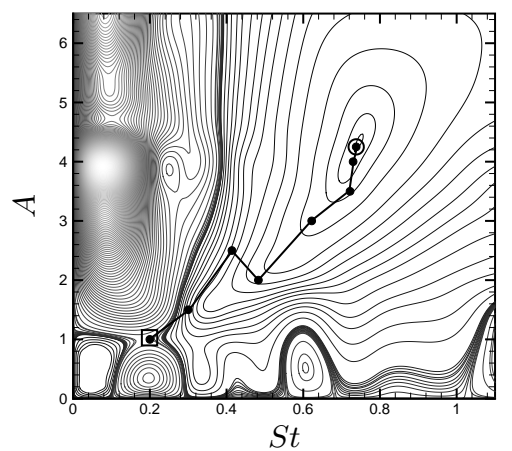

(a) $\boldsymbol{c}_{0}=(1.0,0.2)^{T}$

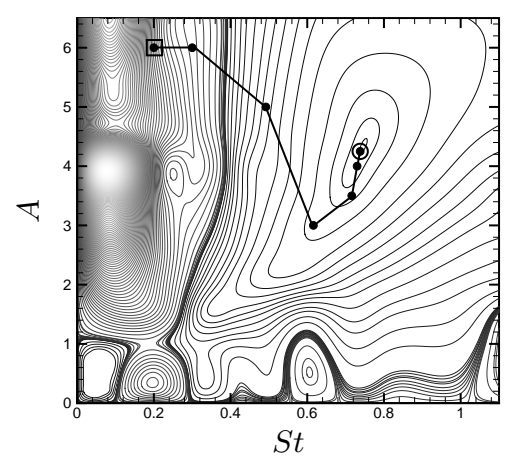

(c) $\boldsymbol{c}_{0}=(6.0,0.2)^{T}$

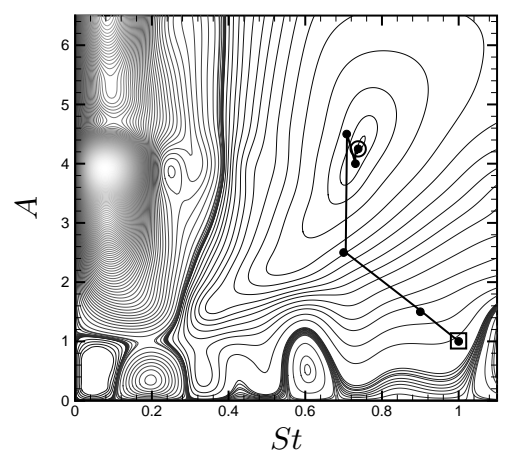

(b) $\boldsymbol{c}_{0}=(1.0,1.0)^{T}$

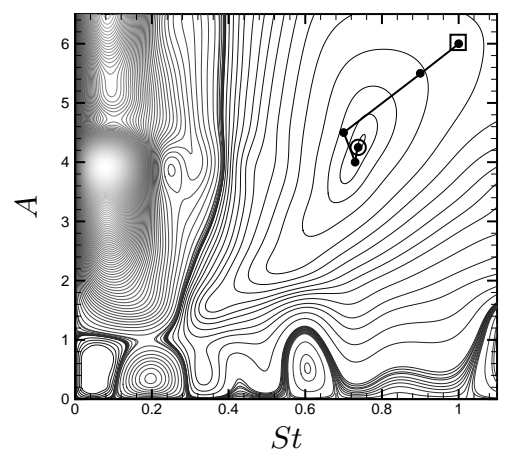

(d) $\boldsymbol{c}_{0}=(6.0,1.0)^{T}$

Figure 6 Evolution of the control parameters during the optimization process for the different initial values $\boldsymbol{c}_{0}$. 
global convergence properties of the TRPOD algorithm $[11,6]$, these optimal control parameters tend towards the values predicted by an open-loop control approach $(\S 1.2)$, and this, whatever the initial values used for the control parameters (see Fig. 6 which represents the convergence in the control parameter space). This proves the performance and the robustness of the TRPOD algorithm. Figure 7(a) represents the time evolutions of the

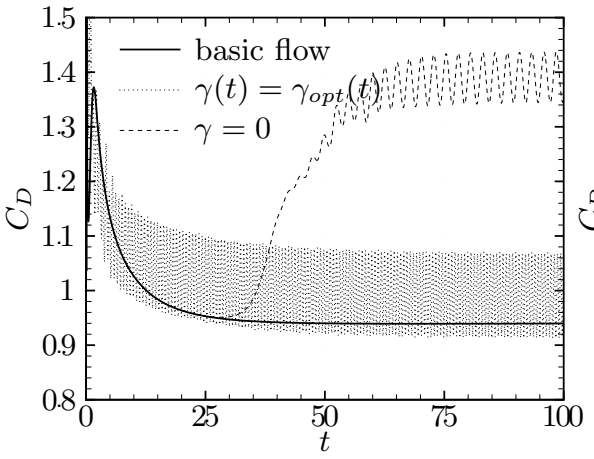

(a) Drag coefficient.

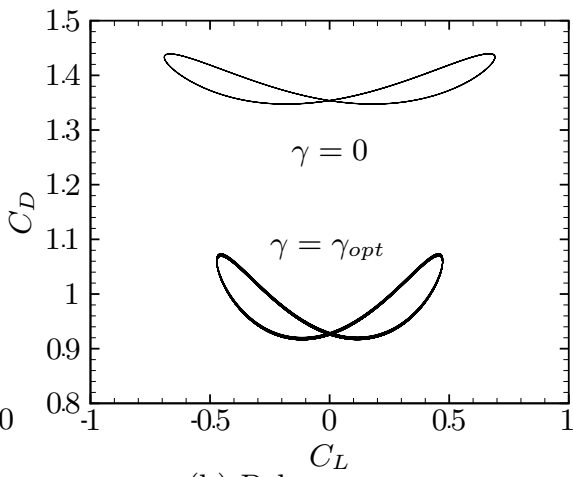

(b) Polar curves.

Figure 7 Time evolutions of the aerodynamic coefficients for the basic flow, uncontrolled flow $(\gamma=0)$ and optimally controlled flow $\left(\gamma(t)=\gamma_{o p t}(t)\right)$. Control was started at time $t=0$.

drag coefficients, for an uncontrolled flow and for the flow forced by the optimal control law $\gamma_{o p t}(t)$. These results are compared to those obtained for the basic flow. According to [8], the basic flow generates a priori the lowest $^{3}$ coefficient of drag for the configuration under study. The mean drag coefficient varies from a value equal to $\left\langle C_{D}{ }^{u n c}\right\rangle_{T}=1.39$ in the uncontrolled case to a value equal to $\left\langle C_{D}{ }^{o p t}\right\rangle_{T}=0.99$ when the optimal control parameters are applied. The corresponding relative mean drag reduction, defined as $\left(\left\langle C_{D}{ }^{u n c}\right\rangle_{T}-\left\langle C_{D}{ }^{\text {opt }}\right\rangle_{T}\right) /\left\langle C_{D}{ }^{u n c}\right\rangle_{T}$, is equal to more than $30 \%$. The value of the drag coefficient for the optimally controlled flow tends towards that obtained for the basic flow $\left(C_{D}^{\text {basic }}=0.94\right)$, but with a value always slightly higher. The polar curves (time evolution of the drag coefficient versus the lift coefficient) are represented for the uncontrolled and optimally controlled flow in Fig. 7(b). The shape of these curves indicates characteristically that, for the two flow regimes, the drag coefficient oscillates at a frequency equal to twice that of the lift coefficient. Finally, in Figs. 8(a)-8(c) we represent the vorticity fields of the uncontrolled flow, the optimally controlled flow,

\footnotetext{
${ }^{3}$ Recently, numerical evidence were brought [19] that, for the circular cylinder wake flow at $R e=200$, a partial control restricted to an upstream part of the cylinder surface could lead to a mean flow correction field with negative drag.
} 
and the basic flow, respectively. The significant vortex-shedding phenomenon observed in Fig. 8(a) has been substantially reduced when the control is applied and the flow has been quasisymmetrized. The resulting flow approaches the symmetric state characteristic of the corresponding basic flow as can be awaited from the results presented in [8] and the discussion in [5]. Our results are qualitatively similar to the effects observed in [20] and [9] and confirm the arguments of [21] that the mean drag reduction is associated with control driving the mean flow toward the unstable state.
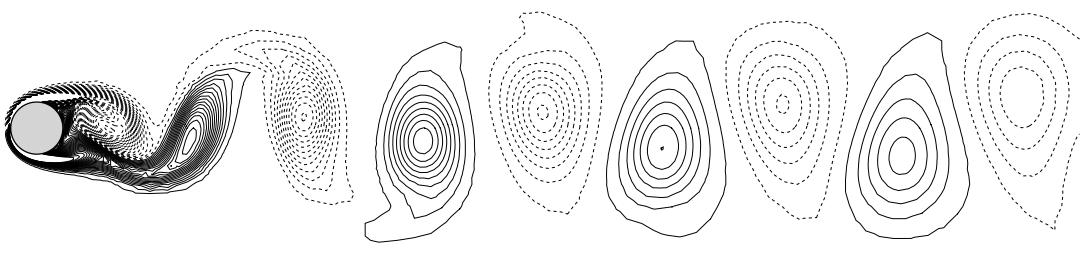

(a) Uncontrolled flow $(\gamma=0)$.

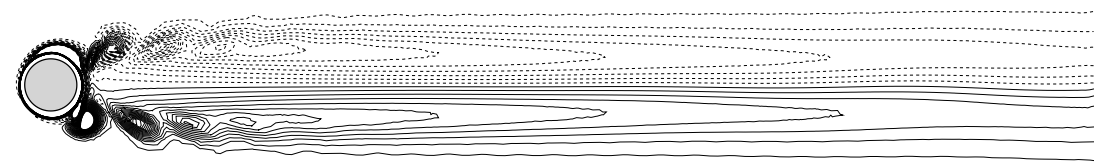

(b) Optimally controlled flow $(\gamma(t)=A \sin (2 \pi S t t)$ with $A=4.25$ and $S t=0.738)$.

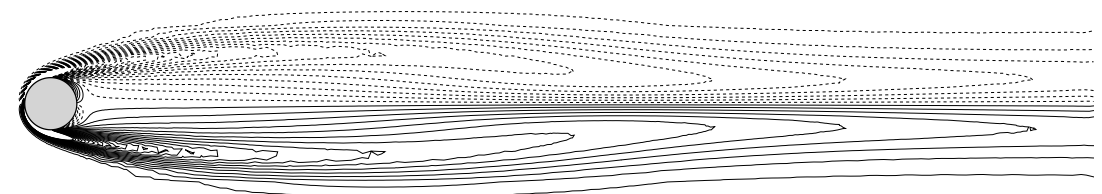

(c) Basic flow $(\gamma=0)$.

Figure 8 Vorticity contour plot of the wake for the uncontrolled (a), optimally controlled (b) and basic flow (c). The dashed lines correspond to negative values. 


\section{Conclusions}

The Trust-Region POD algorithm originally introduced in [6] was used to minimize the total mean drag coefficient of a circular cylinder wake flow in the laminar regime $(R e=200)$. The key enablers to an accurate and robust POD ROM for the pressure and velocity fields were the extension of the POD basis functions to the pressure data, the introduction of a timedependent eddy-viscosity estimated for each POD mode as the solution of an auxiliary optimization problem, and the inclusion in the POD ROM of different non-equilibrium modes. Finally, the optimal control parameters obtained with the TRPOD algorithm are $A=4.25$ and $S t_{f}=0.738$. The relative mean drag reduction is equal to $30 \%$. Compared to previous similar studies where the Navier-Stokes equations are used as state equations in the optimal control problem, the main advantage of the TRPOD algorithm is that it leads to a significant reduction of the numerical costs because the optimization process itself is completely based on reduced-order models only. Indeed, when the state equations of the optimality system are POD ROMs instead of the Navier-Stokes equations, a cost reduction factor of 1600 is obtained for the memory and the optimization problem is solved approximately 4 times more quickly. Now, if we compare to our preceding study [5], where a POD ROM was coupled to an optimal control approach without any strategy for updating the reduced-order model during the optimization process, the cost reduction factors, found here, are lower. However, in this study, the use of the TRPOD algorithm mathematically proves that the solutions converge at least to a local optimum for the original high-fidelity problem, and less than ten resolutions of the Navier-Stokes equations are necessary. Due to the low computational costs involved in the optimization process and the mathematical proofs of global convergence, the TRPOD algorithm is a promising method of optimization in flow control. This approach that can easily be adapted to other configurations, should finally lead to the current resolution of unsteady, three-dimensional optimization problems for turbulent flows around complex geometries.

\section{Acknowledgments}

The authors acknowledge M. Braza (Institut de Mécanique des Fluides de Toulouse) and D. Ruiz (ENSEEIHT) to kindly provide us with an original version of their Matlab Navier-Stokes solver. Stimulating and fruitful discussions with the low-dimensional modelling and control team at the Technische Universität Berlin, in particular Bernd R. Noack, are acknowledged. 


\section{References}

[1] Gunzburger, M.: Flow control. Springer, New York (1995)

[2] Gunzburger, M.: Introduction into mathematical aspects of flow control and optimization. In: Lecture series 1997-05 on inverse design and optimization methods. Von Kármán Institute for Fluid Dynamics (1997)

[3] Cordier, L., Bergmann, M.: Proper Orthogonal Decomposition: an overview. In: Lecture series 2002-04 on post-processing of experimental and numerical data. Von Kármán Institute for Fluid Dynamics (2002)

[4] Cordier, L., Bergmann, M.: Two typical applications of POD: coherent structures eduction and reduced order modelling. In: Lecture series 2002-04 on post-processing of experimental and numerical data. Von Kármán Institute for Fluid Dynamics (2002)

[5] Bergmann, M., Cordier, L., Brancher, J.P.: Optimal rotary control of the cylinder wake using POD Reduced Order Model. Phys. Fluids 17 (2005) 097101:1-21

[6] Fahl, M.: Trust-Region methods for flow control based on Reduced Order Modeling. PhD thesis, Trier university (2000)

[7] Williamson, C.: Vortex dynamics in the cylinder wake. Ann. Rev. Fluid. Mech. 28 (1996) 477-539

[8] Protas, B., Wesfreid, J.: Drag force in the open-loop control of the cylinder wake in the laminar regime. Phys. Fluids 14 (2002) 810-826

[9] He, J.W., Glowinski, R., Metcalfe, R., Nordlander, A., Périaux, J.: Active control and drag optimization for flow past a circular cylinder. Part 1. Oscillatory cylinder rotation. J. Comp. Phys. 163 (2000) 83-117

[10] Homescu, C., Navon, I., Li, Z.: Suppression of vortex shedding for flow around a circular cylinder using optimal control. Int. J. Numer. Meth. Fluids 38 (2002) 43-69

[11] Bergmann, M., Cordier, L.: Control of the cylinder wake in the laminar regime by Trust-Region methods and POD Reduced Order Models. Soumis à J. Fluid Mech. (2006)

[12] Conn, A., Gould, N., Toint, P.: Trust-region methods. SIAM, Philadelphia (2000)

[13] Noack, B., Afanasiev, K., Morzyński, M., Tadmor, G., Thiele, F.: A hierarchy of low-dimensional models for the transient and post-transient cylinder wake. J. Fluid Mech. 497 (2003) 335-363

[14] Galletti, B., Bruneau, C.H., Zannetti, L., Iollo, A.: Low-order modelling of laminar flow regimes past a confined square cylinder. J. Fluid Mech. 503 (2004) 161-170

[15] Bergmann, M.: Optimisation aérodynamique par réduction de modèle POD et contrôle optimal. Application au sillage laminaire d'un cylindre circulaire. $\mathrm{PhD}$ thesis, Institut National Polytechnique de Lorraine, Nancy, France (2004)

[16] Graham, W., Peraire, J., Tang, K.: Optimal Control of Vortex Shedding Using Low Order Models. Part 1. Open-Loop Model Development. Int. J. for Numer. Meth. in Engrg. 44 (1999) 945-972

[17] Nocedal, J., Wright, S.: Numerical Optimization. Springer series in operations research (1999)

[18] Bewley, T.: Flow control: new challenges for a new Renaissance. Progress in Aerospace Sciences 37 (2001) 21-58 
[19] Bergmann, M., Cordier, L., Brancher, J.P.: On the generation of a reverse Von Kármán street for the controlled cylinder wake in the laminar regime. Phys. Fluids 18 (2006) 028101:1-4

[20] Tokumaru, P., Dimotakis, P.: Rotary oscillatory control of a cylinder wake. J. Fluid Mech. 224 (1991) 77-90

[21] Protas, B., Styczek, A.: Optimal rotary control of the cylinder wake in the laminar regime. Phys. Fluids 14 (2002) 2073-2087 